\title{
A Constitutive Formulation for the Linear Thermoelastic Behavior of Arbitrary Fiber-Reinforced Composites
}

\author{
Melek Usal \\ Department of Manufacturing Engineering, Süleyman Demirel University, 32260 Isparta, Turkey \\ Correspondence should be addressed to Melek Usal, esma@mmf.sdu.edu.tr
}

Received 24 November 2009; Revised 20 July 2010; Accepted 14 October 2010

Academic Editor: Horst Ecker

Copyright (C) 2010 Melek Usal. This is an open access article distributed under the Creative Commons Attribution License, which permits unrestricted use, distribution, and reproduction in any medium, provided the original work is properly cited.

The linear thermoelastic behavior of a composite material reinforced by two independent and inextensible fiber families has been analyzed theoretically. The composite material is assumed to be anisotropic, compressible, dependent on temperature gradient, and showing linear elastic behavior. Basic principles and axioms of modern continuum mechanics and equations belonging to kinematics and deformation geometries of fibers have provided guidance and have been determining in the process of this study. The matrix material is supposed to be made of elastic material involving an artificial anisotropy due to fibers reinforcing by arbitrary distributions. As a result of thermodynamic constraints, it has been determined that the free energy function is dependent on a symmetric tensor and two vectors whereas the heat flux vector function is dependent on a symmetric tensor and three vectors. The free energy and heat flux vector functions have been represented by a power series expansion, and the type and the number of terms taken into consideration in this series expansion have determined the linearity of the medium. The linear constitutive equations of the stress and heat flux vector are substituted in the Cauchy equation of motion and in the equation of conservation of energy to obtain the field equations.

\section{Introduction}

Generally, composite materials are separated into natural composites and artificial composites. While natural and artificial composites have functional similarities, they differ greatly in terms of methods of production and purposes of use. Natural composites are not the result of a manufacturing and production method implemented by humans for a certain purpose. Having extremely fine and complex subsystems, structures of this kind comprise known natural structure elements by combination in a certain distribution at a time and on grounds determined according to a universal program of sometimes microscopic and other times macroscopic level. Artificial composites appear as a product of a certain manufacturing 
process produced by intellectual capabilities of the human mind to create a material with superior characteristics for a certain application purpose. Dealing with microlevel study for natural and artificial composite structure elements is a subject of micromechanics [1].

Generally, studies of composite materials are divided into two main branches, namely, micromechanical and macromechanical analyses. The micromechanical analysis aims to uncover certain mechanical characteristics relating to the general behavior of composite materials using the physical and mechanical properties of matrix and reinforcement materials as a starting point. Micromechanical methods can be separated into three, that is, the energy method, elasticity method, and material mechanics method [2]. Composites are broadly used in civilian and military aircraft, aerospace technologies, automotive industry, sea vehicles primarily in ships, pneumatic vessels, power transmission axles, and orthopedic devices [3].

In our previous study [4], viscoelastic composites of a single fiber family have been studied assuming that the medium has a discontinuity surface. In our study [5], it has been assumed that a viscoelastic medium with two different inextensible fiber families does not have a discontinuity surface. Again, in the studies in [6,7], the exposure of a viscoelastic medium to the effect of electrical and magnetic fields in addition to its reinforcement by a single fiber family has been researched in the form of separate studies. Furthermore, in his study [8], Usal has examined the electromechanical behavior of a piezoelectric viscoelastic medium with two fiber families. Since temperature was assumed to be constant in all of the previous studies, temperature change has not been taken into consideration. In this study, constitutive equations have been obtained that indicate the stress and heat distribution determining the thermoelastic behavior of a composite material reinforced by two arbitrary independent and inextensible fiber families. Since the temperature is not constant, a temperature gradient has been included in the calculations as an independent constitutive variable.

Researchers like those in [9-11] have made progress in the studies they have conducted on the formulation of thermoelastic effect on a variety of materials. A study by Nowacki fills a large gap in thermoelasticity and its applications [12].

The thermal properties can play a significant role in affecting the design and manufacture of composite structures in their industrial applications [13]. The subject of thermoelastic behavior of composites has been studied by a number of different researchers [14-21]. Fiber-reinforced composite materials belong to a very important class of materials which are often employed in a wide variety of industrial applications. Typically, these composite materials consist of a fabric structure where the fibers are continuously arranged in a matrix material, and, at the macroscopic level, these composite materials exhibit strong directional dependencies. The vehicle tyres furnishe a typical example of technological application of such man-made composites [21].

Due to some technological requirements, it is aspired that specific construction elements have rather elastic properties, provided that they have high durability in certain directions. Fiber-reinforced composite materials are produced by sticking fibers in a polymeric matrix which is elastic but with low strength. These fibers are manufactured from high-strength graphite or bor. They can be easily bent due to the very small size of their cross-section and it can be assumed that these fibers show a continuous distribution in a medium. Assuming inextensibility of the fibers is a reasonable approach since the rigidity of the fibers is very high compared to the rigidity of the matrix. Inextensibility of the fibers is broadly accepted in practice for formulation purposes. Thus, fiber families are assumed to be inextensible $\left(\lambda_{a}^{2}=C_{K L} A_{K} A_{L}=1, \lambda_{z}^{2}=C_{K L} Z_{K} Z_{L}=1\right)$ [22]. On the other hand, the composite material taken into consideration in this work is assumed to be compressible and shows 
linear elastic behavior. In a class of engineered fiber composites for structural load-bearing components in civil or aerospace applications, an assumption of linear elastic behavior is suitable and this class of composites belongs to a compressible material response.

\section{Kinematics of Fibers Deformation}

It is assumed that an element from two different continuous fiber families is placed on each point of the composite material. Before deformation and after deformation, these fiber families are represented by continuous unit vectors $\mathbf{A}(\mathbf{X}), \mathbf{Z}(\mathbf{X}), \mathbf{a}(\mathbf{x})$, and $\mathbf{z}(\mathbf{x})$, respectively. The fibers deform along with the material; that is, fibers do not have a relative motion with respect to the material in which they are embedded. Relationships given below are true for an A-fiber family $[23,24]$

$$
a_{k} d l=x_{k, K} A_{K} d L, \quad a_{k} \frac{d l}{d L}=x_{k, K} A_{K} .
$$

Rates of extension of fiber family $A$ can be defined as follows:

$$
\lambda_{a} \equiv\left(\frac{d l}{d L}\right)_{A}
$$

If expression (2.2) is substituted into (2.1), the following expression is obtained:

$$
a_{k}=\lambda_{a}^{-1} x_{k, K} A_{K}
$$

Deformation geometry of the fiber family $A$ is expressed by relationship (2.3). Because vectors $\mathbf{A}$ and a here represent unitized vectors of the fiber family $A$, operations are true

$$
|\mathbf{A}|=|\mathbf{a}|=1, \quad a_{k} a_{k}=1=\lambda_{a}^{-2} x_{k, K} x_{k, L} A_{K} A_{L}=\lambda_{a}^{-2} C_{K L} A_{K} A_{L} .
$$

Accordingly, the form is found to be

$$
\lambda_{a}^{2}=C_{K L} A_{K} A_{L} .
$$

Relationships that are true for the Z-fiber family can be expressed as follows [23, 24]:

$$
z_{k}=\lambda_{z}^{-1} x_{k, K} Z_{K}, \quad \lambda_{z} \equiv\left(\frac{d l}{d L}\right)_{Z}, \quad \lambda_{z}^{2}=C_{K L} Z_{K} Z_{L}
$$

where $d L$ and $d l$ are, respectively, arc length of fiber before and after deformation, $A_{K}$ and $Z_{K}$ are fiber unit vector components before deformation, $a_{k}$ and $z_{k}$ are fiber unit vector components after deformation, $x_{k, K}=\partial x_{k} / \partial X_{K}$ is deformation gradient, $\lambda_{a}$ and $\lambda_{z}$ are rates of extension of fiber families, and $C_{K L}=x_{k, K} x_{k, L}$ is Green deformation tensor. 


\section{Thermomechanic Balance Equations}

Balance equations, mass, linear momentum, angular momentum, energy balances, and entropy inequality have been summarized in $[22,25]$. We have the following:

Conservation of mass:

$$
\dot{\rho}+\rho v_{k, k}=0, \quad \rho(\mathbf{x}, t)=\frac{\rho_{0}(\mathbf{X})}{J(\mathbf{x}, t)} \text { (conservation of mass in material representation), }
$$

balance of linear mometum:

$$
\rho \dot{v}_{p}=\rho \mathrm{f}_{p}+t_{r p, r}
$$

balance of moment of momentum:

$$
\varepsilon_{k r p} t_{r p}=0, \quad t_{r p}=t_{p r}
$$

conservation of energy:

$$
\rho \dot{\varepsilon}=t_{k l} d_{k l}+q_{k, k}+\rho h
$$

Clausius-Duhem inequality:

$$
\rho \theta \dot{\eta}-\nabla \cdot \mathbf{q}+\frac{1}{\theta} \mathbf{q} \cdot \nabla \theta-\rho \mathbf{h} \geqslant 0
$$

Here, $v$ stands for the velocity field in a continuous medium, $\rho_{0}$ for mass density before deformation, $\rho$ for mass density after deformation, $J \equiv \operatorname{det}\left[x_{k, K}\right]=\rho_{0} / \rho(\mathbf{x}, t)$ for jacobian, $\dot{v}$ for acceleration, $t_{l k}$ for stress tensor, $\mathrm{f}_{k}$ for the mechanical volumetric force per unit of mass, $\varepsilon$ for internal energy density per unit of mass, $q_{k}$ for heat flux vector, $h$ for heat source per unit of mass, $\eta$ for entropy density per unit of mass, $\theta(\mathbf{X}, t)$ for the absolute temperature of a material point $\mathbf{X}$ at a moment $t$, and $\varepsilon_{i j k}$ for permutation tensor.

\section{Thermodynamic Constraints and Modeling Constitutive Equations}

Taking ( $\rho h)$ from the local energy (3.4) and substituting it in the entropy inequality (3.5) will give us the following:

$$
-\rho(\dot{\varepsilon}-\theta \dot{\eta})+t_{k l} d_{l k}+\frac{1}{\theta} q_{k} \theta_{, k} \geq 0 .
$$


Since the material derivative of the entropy density in this expression cannot be controlled inside a thermodynamic process, a defined Legendre transformation like the one provided below can be used to transform the derivative of these values into the controllable value $\theta$

$$
\psi \equiv \varepsilon-\theta \eta
$$

As a result, the entropy inequality is transformed as follows, expressed in new terms:

$$
-\rho(\dot{\psi}+\dot{\theta} \eta)+t_{k l} d_{l k}+\frac{1}{\theta} q_{k} \theta_{, k} \geq 0
$$

Entropy inequality is obtained as follows in the material form [26]:

$$
-\left(\dot{\Sigma}+\rho_{0} \dot{\theta} \eta\right)+\frac{1}{2} T_{K L} \dot{C}_{K L}+\frac{1}{\theta} \theta_{, K} Q_{K} \geq 0
$$

Terms relating to the new values have been provided below:

$$
\begin{gathered}
\Sigma \equiv \rho_{0} \psi, \\
\dot{C}_{K L}=2 \mathrm{~d}_{k 1} x_{k, K} x_{1, L} \Longrightarrow d_{k l}=\frac{1}{2} \dot{C}_{K L} X_{K, k} X_{L, l}, \\
T_{K L} \equiv J X_{K, k} X_{L, l} t_{k l} \Longrightarrow t_{k l}=J^{-1} x_{k, K} x_{l, L} T_{K L}, \\
Q_{K} \equiv J X_{K, k} q_{k} \Longrightarrow q_{k}=J^{-1} x_{k, K} Q_{K}, \\
G_{K} \equiv \theta_{, K}=x_{k, K} \theta_{, k} \Longrightarrow g_{k} \equiv \theta_{, k}=X_{K, k} \theta_{, K} .
\end{gathered}
$$

Here, $\Sigma$ stands for thermodynamic stress potential, $\psi$ for generalized free energy density, $d_{k l}$ for deformation (strain) rate tensor, $X_{K, k}=\partial X_{K} / \partial x_{k}$ for the deformation gradient of the reverse motion, $T_{K L}$ for the stress tensor on material coordinates, $Q_{K}$ for the heat flux vector on material coordinates, and $G_{K}$ for the temperature gradient on material coordinates.

To be able to use the inequality (4.4), we need to know the independent variables on which the thermodynamic potential $\Sigma$ depends. Arguments of $\Sigma$ and the variables they depend on have been found using constitutive axioms based on the selected material. According to the axioms of causality and determinism [22, 25], our stress potential, as a response functional at a material point $X$ at a time $t$, can be written as follows:

$$
\Sigma(\mathbf{X}, t)=\Sigma\left[\mathbf{x}\left(\mathbf{X}^{\prime}, t^{\prime}\right), \theta\left(\mathbf{X}^{\prime}, t^{\prime}\right), \mathbf{X}\right] \quad X \in V-\infty<t^{\prime} \leq t .
$$

Here, $t^{\prime}$ is any point in time between now and the past. $\mathbf{X}^{\prime}$ stands for all material points other than $\mathbf{X}$.

Using the results of causality, determinism, objectivity, smooth neighborhood, and admissibility axioms $[22,25]$, the arguments on which $\Sigma$ depends in a composite with two 
fiber families exposed to mechanical loading and temperature change can be expressed as follows:

$$
\Sigma\left(X_{K}, t\right)=\Sigma\left[C_{K L}\left(X_{K}, t\right), G_{K}\left(X_{K}, t\right), A_{K}\left(X_{K}\right), Z_{K}\left(X_{K}\right), \theta\left(X_{K}, t\right), X_{K}\right] .
$$

Assuming that the materials are homogenous, $\mathbf{X}$ will be eliminated from among the arguments given in the expression (4.11) on which $\Sigma$ depends. Because the fiber vectors $A_{K}$ and $Z_{K}$ do not depend on time, the following expression is obtained by taking the material derivative of expression (4.11).

$$
\dot{\Sigma}=\frac{\partial \Sigma}{\partial C_{K L}} \dot{C}_{K L}+\frac{\partial \Sigma}{\partial \theta_{, K}} \dot{G}+\frac{\partial \Sigma}{\partial \theta} \dot{\theta}
$$

Substituting this expression in (4.4) gives us the following inequality:

$$
\frac{1}{2}\left(T_{K L}-2 \frac{\partial \Sigma}{\partial C_{K L}}\right) \dot{C}_{K L}-\rho_{0}\left(\eta+\frac{1}{\rho_{0}} \frac{\partial \Sigma}{\partial \theta}\right) \dot{\theta}-\frac{\partial \Sigma}{\partial G_{K}} \dot{G}_{K}+\frac{1}{\theta} G_{K} Q_{K} \geq 0
$$

Since we are able to arbitrarily replace the arguments in inequality (4.13) from $\theta$ to $\dot{\theta}$, from $C_{K L}$ to $\dot{C}_{K L}$, and from $G_{K}$ to $\dot{G}_{K}$, for the inequality (4.13) to be satisfied, the coefficients of $\dot{\theta}$, $\dot{C}_{K L}$ and $\dot{G}_{K}$ will be zero. The coefficient of $G_{K}$ cannot be zero as due to $G_{K}$ 's presence in the arguments of $\Sigma$, it cannot be arbitrarily replaced. Equalizing the coefficients of $\dot{C}_{K L}, \dot{\theta}$, and $\dot{G}_{K}$ to zero will give us the following expressions:

$$
\begin{gathered}
T_{K L}=2 \frac{\partial \Sigma}{\partial C_{K L}}, \\
\eta=-\frac{1}{\rho_{0}} \frac{\partial \Sigma}{\partial \theta} \\
\frac{\partial \Sigma}{\partial G_{K}}=0 .
\end{gathered}
$$

It is understood from expression (4.16) that the stress potential does not depend on $G_{K}$. Therefore, arguments on which the stress potential depends are expressed as follows:

$$
\Sigma=\Sigma\left(C_{K L}, A_{K}, Z_{K}, \theta\right)
$$

Thus, inequality (4.13) is reduced to the following form:

$$
\frac{1}{\theta} G_{K} Q_{K} \geq 0
$$

For the heat flux vector, expression (4.18) gives the Clausius-Duhem inequality and the following expression indicates the arguments on which the heat flux vector depends

$$
Q_{K}=Q_{K}\left(C_{K L}, A_{K}, Z_{K}, G_{K}, \theta\right)
$$


In consideration of expression (4.19), inequality (4.18) is written down as follows:

$$
G_{K} Q_{K}\left(C_{K L}, A_{K}, Z_{K}, G_{K}, \theta\right) \geq 0 \text { or } \mathbf{Q}(\mathbf{C}, \mathbf{A}, \mathbf{Z}, \mathbf{G}, \theta, \mathbf{X}) \cdot \mathbf{G} \geq 0 .
$$

In inequality (4.20), when $G_{K}=0, Q_{K}$ must also be equal to zero. Accordingly, maintaining the order of independent constitutive variables in expression (4.20), the following expression should be written:

$$
Q_{K}\left(C_{K L}, A_{K}, Z_{K}, 0, \theta\right)=0 .
$$

On the other hand, internal energy density $\varepsilon$ can be written as follows from the expressions (4.2), (4.5), and (4.15):

$$
\varepsilon=\frac{1}{\rho_{0}}\left(\Sigma-\frac{\partial \Sigma}{\partial \theta} \theta\right)
$$

From the constitutive equations offered by expressions (4.14) and (4.19), it is understood that the stress is derived from the stress potential $\Sigma$, while the heat flux vector appears as a vectorial form with known arguments independent of the stress potential. Thus, the explicit forms of $\Sigma$ and $Q_{K}$, which appear as constitutive functions with definite arguments, should be determined. However, constraints imposed on the constitutive functions of the material in question by the material symmetry axiom should firstly be revised.

Let the symmetry group of the material be the full orthogonal group (isotropic material) or any of its subgroups (anisotropic material). Let $\underline{\underline{S}}=\left[S_{K L}\right]$ be any arbitrary matrix representing the orthogonal transformation of material coordinates (or rigid configurations of the material medium according to the reference coordinate frame) and pertaining to the symmetry group of the medium. According to the material symmetry axiom, constitutive functionals under each transformation

$$
X_{K}^{\prime}=S_{K L} X_{L}, \quad X_{L}=S_{L K}^{T} X_{K}^{\prime}, \quad \underline{\underline{S}}^{-1}=\underline{\underline{S}}^{T}
$$

established using the orthogonal matrix $\underline{\underline{S}}$ should remain form invariant. Mathematically, this indicates the validity of the transformations

$$
\begin{gathered}
\Sigma\left(\underline{\underline{S}} \underline{\underline{C}} \underline{\underline{S}}^{T}, \underline{\underline{S}} \underline{\underline{A}}, \underline{\underline{S}} \underline{\underline{Z}}, \theta\right)=\Sigma(\underline{\underline{C}}, \underline{A}, \underline{Z}, \theta), \\
\underline{Q}\left(\underline{\underline{S}} \underline{\underline{C}} \underline{\underline{S}}^{T}, \underline{\underline{S}} \underline{A}, \underline{\underline{S}} \underline{\underline{Z}}, \underline{\underline{S}} \underline{G}, \theta\right)=\underline{Q}(\underline{\underline{C}}, \underline{A}, \underline{Z}, \underline{G}, \theta) .
\end{gathered}
$$

The following conditions should be satisfied since the fiber families are assumed to be inextensible [22, 27]:

$$
\lambda_{a}^{2}=C_{K L} A_{K} A_{L}=1, \quad \lambda_{z}^{2}=C_{K L} Z_{K} Z_{L}=1 .
$$


Thus, the constitutive equation for the stress is obtained as follows in spatial and material coordinates:

$$
\begin{aligned}
t_{k l} & =\Gamma_{a} a_{k} a_{l}+\Gamma_{z} z_{k} z_{l}+2 x_{k, K} x_{l . L} \frac{\partial \Sigma}{\partial C_{K L}}, \\
T_{K L} & =\Gamma_{a} A_{K} A_{L}+\Gamma_{z} Z_{K} Z_{L}+2 \frac{\partial \Sigma}{\partial C_{K L}} .
\end{aligned}
$$

In these expressions, $\Gamma_{a}$ and $\Gamma_{z}$ are Lagrange coefficients and are defined by field equations and boundary conditions.

In this study, the matrix material has been considered as an anisotropic medium. In the scope of this approach, the stress potential and heat flux vector functions are expanded in power series in terms of the components of arguments on which they depend, giving us the thermoelastic behavior of the composite medium. The reference position of the medium has been selected at a uniform temperature $T_{0}$ in a stress-free natural condition, and it has been assumed that the medium moves away from that position by small displacements and deformations and small changes in temperature. By referring to small changes in temperature, we mean $\theta=T_{0}+T, T_{0}>0,|T| \ll T_{0}[22]$. The type and the number of terms taken in the series expansion have been determined based on the linearity condition of the medium. Moreover, because the matrix material remains insensitive to change of direction along the fibers, expressions of vector fields representing the fiber distribution through outer products in even numbers as arguments should be considered. The linear constitutive equation of stress has been obtained by taking the derivative of the stress potential according to its deformation tensor. Field equations have been obtained by substituting the linear constitutive equations of the stress and heat flux vector in the Cauchy motion equation and in the equation of conservation of energy.

\section{Determination of Stress Constitutive Equation in Linear Thermoelasticity}

Since the relation $C_{K L}=\delta_{K L}+2 E_{K L}$ exists between the Green deformation tensor and strain tensor, $E_{K L}$ can be expressed as $E_{K L} \cong \widetilde{E}_{K L} \equiv(1 / 2)\left(U_{K, L}+U_{L, K}\right)$ in a linear theory, and the arguments of the stress potential given by expression (4.17) can be written down as follows:

$$
\Sigma=\Sigma\left(\widetilde{E}_{K L}, A_{K}, Z_{K}, \theta\right)
$$

Assuming that this function is analytic in terms of the $\widetilde{\mathbf{E}}, \mathbf{A}, \mathbf{Z}$ values, if this function is expanded in Taylor series around $\widetilde{\mathbf{E}}=\mathbf{0}, \mathbf{A}=\mathbf{0}, \mathbf{Z}=\mathbf{0}$, the expression will be obtained for the stress potential:

$$
\begin{aligned}
\Sigma\left(\tilde{E}_{K L}, A_{S}, Z_{Y}, \theta\right)= & \Sigma_{0}(\theta, \mathbf{X})+\Sigma_{K L}(\theta, \mathbf{X}) \tilde{E}_{K L}+\frac{1}{2} \Sigma_{K L M N}(\theta, \mathbf{X}) \tilde{E}_{K L} \tilde{E}_{M N}+\lambda_{S N}(\theta, \mathbf{X}) A_{S} A_{N} \\
& +\Omega_{Y N}(\theta, \mathbf{X}) Z_{Y} Z_{N}+\zeta_{K L S N}(\theta, \mathbf{X}) E_{K L} A_{S} A_{N} \\
& +\kappa_{K L Y N}(\theta, \mathbf{X}) E_{K L} Z_{Y} Z_{N}+\cdots
\end{aligned}
$$


Coefficients in this equation have been defined as follows:

$$
\begin{aligned}
& \Sigma_{0}=\Sigma(\underline{\underline{0}}, \underline{0}),\left.\quad \Sigma_{K L} \equiv \frac{\partial \Sigma}{\partial \widetilde{E}_{K L}}\right|_{0},\left.\quad \Sigma_{K L M N} \equiv \frac{\partial^{2} \Sigma}{\partial \widetilde{E}_{K L} \partial \widetilde{E}_{M N}}\right|_{0},\left.\quad \lambda_{S N} \equiv \frac{1}{2} \frac{\partial^{2} \Sigma}{\partial A_{S} \partial A_{N}}\right|_{0} ^{\prime} \\
& \left.\Omega_{Y N} \equiv \frac{1}{2} \frac{\partial^{2} \Sigma}{\partial Z_{Y} \partial Z_{N}}\right|_{0},\left.\left.\quad \zeta_{K L S N} \equiv \frac{1}{6} \frac{\partial^{3} \Sigma}{\partial E_{K L} \partial A_{S} \partial A_{N}}\right|_{0} ^{,} \quad \kappa_{K L Y N} \equiv \frac{1}{6} \frac{\partial^{3} \Sigma}{\partial E_{K L} \partial Z_{Y} \partial Z_{N}}\right|_{0} .
\end{aligned}
$$

Due to the symmetry of the $\widetilde{E}_{K L}$ tensor and nondependence on order of the derivatives in the definitions in the expressions (5.3), these coefficients bear the symmetry characteristics indicated below:

$$
\begin{gathered}
\Sigma_{K L}=\Sigma_{L K}, \quad \Sigma_{K L M N}=\Sigma_{L K M N}=\Sigma_{K L N M}=\Sigma_{M N K L}, \quad \lambda_{S N}=\lambda_{N S}, \quad \Omega_{Y N}=\Omega_{N Y}, \\
\zeta_{K L S N}=\zeta_{L K S N}=\zeta_{K L N S}, \quad \kappa_{K L Y N}=\kappa_{L K Y N}=\kappa_{K L N Y} .
\end{gathered}
$$

The following relations can be written down for the linear theory in continuum mechanics [22]:

$$
\begin{gathered}
E_{K L} \cong \widetilde{E}_{K L} \equiv \frac{1}{2}\left(U_{K, L}+U_{L, K}\right), \quad e_{k l} \cong \tilde{e}_{k l}=\epsilon_{k l} \equiv \frac{1}{2}\left(u_{k, l}+u_{l, k}\right), \quad \in_{k l} \cong \lambda_{k K} \lambda_{l L} \widetilde{E}_{K L}, \\
\tilde{E}_{K L} \cong \lambda_{k K} \lambda_{l L} \tilde{e}_{k l}, \quad x_{k, K}=\lambda_{k K}+u_{k, K}, \quad X_{K, k}=\Lambda_{K k}-U_{K, k}, \quad x_{k, K} x_{l, L}=\lambda_{k K} \lambda_{l L} \\
X_{K, k} X_{L, l}=\lambda_{k K} \lambda_{l L}, \quad E_{K L} \cong \widetilde{E}_{K L} \equiv \lambda_{k K} \lambda_{l L} \tilde{e}_{k l}=\frac{1}{2} \lambda_{k K} \lambda_{l L}\left(u_{k, l}+u_{l, k}\right), \\
x_{p, P} x_{r, R} A_{K} A_{L}=x_{p, P} x_{r, R} X_{K, k} X_{L, l} a_{k} a_{l} \lambda_{a}^{2}=\lambda_{p P} \lambda_{r R} \lambda_{k K} \lambda_{l L} a_{k} a_{l} \quad\left(\text { for } \lambda_{a}=1\right), \\
x_{p, P} x_{r, R} Z_{K} Z_{L}=x_{p, P} x_{r, R} X_{K, k} X_{L, l} z_{k} z_{l} \lambda_{z}^{2}=\lambda_{p P} \lambda_{r R} \lambda_{k K} \lambda_{l L} z_{k} z_{l} \quad\left(\text { for } \lambda_{z}=1\right), \\
d_{p r}=\frac{\partial E_{P R}}{\partial t} X_{P, r} X_{R, r}=\frac{\partial \epsilon_{p r}}{\partial t}, \quad d_{p r}=\frac{\partial\left(u_{p, r}\right)}{\partial t}, \quad \dot{\varepsilon} \approx \frac{\partial \varepsilon}{\partial t} \\
J^{-1} \cong 1-u_{k, k}, \quad \rho \cong \rho_{0}\left(1-u_{k, k}\right) .
\end{gathered}
$$

The expression of the spatial form of stress for compressible media with inextensible fiber families can be written down as indicated below:

$$
t_{p r}=\Gamma_{a} a_{k} a_{l}+\Gamma_{z} z_{k} z_{l}+\left(1-u_{k, k}\right) \frac{\partial \Sigma}{\partial \epsilon_{p r}}
$$

In the linear theory, arguments on which $\Sigma$ depends can be expressed in spatial form as follows:

$$
\Sigma=\Sigma\left(\epsilon_{k l}, a_{k}, z_{k}, \theta, \mathbf{X}\right)
$$


Assuming this function is analytic in terms of $\epsilon_{k l}, a_{k}, z_{k}$ and expanding it in the Taylor series around $\epsilon_{k l}=0, a_{s}=0, z_{y}=0$ will give us the following expression:

$$
\begin{aligned}
\Sigma\left(\epsilon_{k l}, a_{s}, z_{y}, \theta, \mathbf{X}\right)= & \Sigma_{0}(\theta, \mathbf{X})+\Sigma_{k l}(\theta, \mathbf{X}) \epsilon_{k l}+\frac{1}{2} \Sigma_{k l m n}(\theta, \mathbf{X}) \epsilon_{k l} \in_{m n}+\lambda_{s n}(\theta, \mathbf{X}) a_{s} a_{n} \\
& +\Omega_{y n}(\theta, \mathbf{X}) z_{y} z_{n}+\zeta_{k l s n}(\theta, \mathbf{X}) \in_{k l} a_{s} a_{n}+\kappa_{k l y n}(\theta, \mathbf{X}) \in_{k l} z_{y} z_{n}+\cdots
\end{aligned}
$$

The spatial material tensors $\Sigma_{k l}, \Sigma_{k l m n}, \lambda_{s n}, \Omega_{y n}, \zeta_{k l s n}$, and $\kappa_{k l y n}$ in (5.8) bear the same properties as the material tensors of the material $\Sigma_{K L}, \Sigma_{K L M N}, \lambda_{S N}, \Omega_{Y N}, \zeta_{K L S N}$, and $\kappa_{K L Y N}$ and are defined as follows:

$$
\begin{gathered}
\Sigma_{k l}=\lambda_{k K} \lambda_{l L} \Sigma_{K L}, \quad \Sigma_{k l m n} \equiv \lambda_{k K} \lambda_{l L} \lambda_{m M} \lambda_{n N} \Sigma_{K L M N}, \quad \lambda_{S n}=\lambda_{S S} \lambda_{n N} \lambda_{S N}, \\
\Omega_{y n}=\lambda_{y Y} \lambda_{n N} \Omega_{Y N}, \quad \zeta_{k l s n} \equiv \lambda_{k K} \lambda_{l L} \lambda_{s S} \lambda_{n N} \zeta_{K L S N}, \quad \kappa_{k l y n} \equiv \lambda_{k K} \lambda_{l L} \lambda_{y Y} \lambda_{n N} \kappa_{K L Y N} .
\end{gathered}
$$

In order to obtain a correct formulation of the linear theory, expression (5.8) should be quadratic at most in terms of the endlessly small expansion tensor $\epsilon_{k l}$ and temperature change T. For this purpose, the coefficients dependent on $\theta$ in the expression (5.8) have been defined as follows, respectively:

$$
\begin{aligned}
\Sigma_{0}(\theta, \mathbf{X}) & =\Sigma_{0}\left(T_{0}+T, \mathbf{X}\right)=\rho_{0}(\mathbf{X}) \psi_{0}\left(T_{0}, \mathbf{X}\right)-\rho_{0}(\mathbf{X}) \eta_{0}\left(T_{0}, \mathbf{X}\right) T-\frac{1}{2} \rho_{0}(\mathbf{X}) \frac{1}{T_{0}} C\left(T_{0}, \mathbf{X}\right) T^{2}+\cdots, \\
\Sigma_{k l}(\theta, \mathbf{X}) & =\gamma_{k l}\left(T_{0}, \mathbf{X}\right)-\beta_{k l}\left(T_{0}, \mathbf{X}\right) T+\cdots, \\
\lambda_{s n}(\theta, \mathbf{X}) & =\Lambda_{s n}\left(T_{0}, \mathbf{X}\right)-\mu_{s n}\left(T_{0}, \mathbf{X}\right) T+\cdots, \\
\Omega_{y n}(\theta, \mathbf{X}) & =\Omega_{y n}\left(T_{0}, \mathbf{X}\right)-\pi_{y n}\left(T_{0}, \mathbf{X}\right) T+\cdots, \\
\Sigma_{k l m n}(\theta, \mathbf{X}) & =\Sigma_{k l m n}\left(T_{0}+T, \mathbf{X}\right)=\Sigma_{k l m n}\left(T_{0}, \mathbf{X}\right), \\
\zeta_{k l s n}(\theta, \mathbf{X}) & =\zeta_{k l s n}\left(T_{0}+T, \mathbf{X}\right)=\zeta_{k l s n}\left(T_{0}, \mathbf{X}\right), \\
\kappa_{k l y n}(\theta, \mathbf{X}) & =\kappa_{k l y n}\left(T_{0}+T, \mathbf{X}\right)=\kappa_{k l y n}\left(T_{0}, \mathbf{X}\right) .
\end{aligned}
$$

Coefficients in this equations have been defined as follows:

$$
\begin{aligned}
& \left.\frac{\partial \psi_{0}(T, \mathbf{X})}{\partial T}\right|_{T=T_{0}} \equiv-\eta_{0}\left(T_{0}, \mathbf{X}\right),\left.\quad \frac{\partial^{2} \psi_{0}(T, \mathbf{X})}{\partial T^{2}}\right|_{T=T_{0}} \equiv-\frac{1}{T_{0}} C\left(T_{0}, \mathbf{X}\right), \\
& \gamma_{k l}\left(T_{0}, \mathbf{X}\right) \equiv \Sigma_{k l}\left(T_{0}, \mathbf{X}\right)=\gamma_{l k}\left(T_{0}, \mathbf{X}\right), \quad \beta_{k l}\left(T_{0}, \mathbf{X}\right) \equiv-\left.\frac{\partial \Sigma_{k l}(T, \mathbf{X})}{\partial T}\right|_{T=T_{0}}=\beta_{l k}\left(T_{0}, \mathbf{X}\right), \\
& \Lambda_{s n}\left(T_{0}, \mathbf{X}\right) \equiv \Lambda_{n s}\left(T_{0}, \mathbf{X}\right), \quad \mu_{s n}\left(T_{0}, \mathbf{X}\right) \equiv-\left.\frac{\partial \lambda_{s n}(T, \mathbf{X})}{\partial T}\right|_{T=T_{0}}=\mu_{n s}\left(T_{0}, \mathbf{X}\right) \\
& \Omega_{y n}\left(T_{0}, \mathbf{X}\right) \equiv \Omega_{n y}\left(T_{0}, \mathbf{X}\right), \quad \pi_{y n}\left(T_{0}, \mathbf{X}\right) \equiv-\left.\frac{\partial \Omega_{y n}(T, \mathbf{X})}{\partial T}\right|_{T=T_{0}}=\pi_{n y}\left(T_{0}, \mathbf{X}\right) .
\end{aligned}
$$


In these expressions, $\psi_{0}\left(T_{0}, \mathbf{X}\right), \eta_{0}\left(T_{0}, \mathbf{X}\right)$, and $C\left(T_{0}, \mathbf{X}\right)$ are scalar; $\gamma_{k l}\left(T_{0}, \mathbf{X}\right), \beta_{k l}\left(T_{0}, \mathbf{X}\right)$, $\Lambda_{s n}\left(T_{0}, \mathbf{X}\right), \mu_{s n}\left(T_{0}, \mathbf{X}\right), \Omega_{y n}\left(T_{0}, \mathbf{X}\right), \pi_{y n}\left(T_{0}, \mathbf{X}\right), \Sigma_{k l m n}\left(T_{0}, \mathbf{X}\right), \zeta_{k l s n}\left(T_{0}, \mathbf{X}\right)$, and $\kappa_{k l y n}\left(T_{0}, \mathbf{X}\right)$ are tensorial material constants, and these coefficients depend on the initial temperature $T_{0}$ of the medium and medium particles in heterogeneous materials. In homogenous materials, the dependence on $\mathbf{X}$ is eliminated. In order to simplify notation, we will not indicate the arguments $\left(T_{0}, \mathbf{X}\right)$ of such coefficients. Substituting the expressions (5.11) and (5.10) in (5.8) gives us the following expression:

$$
\begin{aligned}
\Sigma\left(\epsilon_{k l}, a_{s}, z_{y}, T_{0}+T, \mathbf{X}\right)= & \rho_{0} \psi_{0}-\rho_{0} \eta_{0} T-\frac{\rho_{0} c}{2 T_{0}} T^{2}+\gamma_{k l} \in_{k l}-\beta_{k l} T \epsilon_{k l}+\Lambda_{s n} a_{s} a_{n} \\
& -\mu_{s n} T a_{s} a_{n}+\Omega_{y n} z_{y} z_{n}-\pi_{y n} T z_{y} z_{n}+\frac{1}{2} \Sigma_{k l m n} \in_{k l} \in_{m n} \\
& +\zeta_{k l s n} \in_{k l} a_{s} a_{n}+\kappa_{k l y n} \epsilon_{k l} z_{y} z_{n}+\cdots .
\end{aligned}
$$

If derivative in (5.6) is taken from (5.12) and used in substitution, the following expression is obtained:

$$
t_{p r}=\Gamma_{a} a_{p} a_{r}+\Gamma_{z} z_{p} z_{r}+\left(1-u_{k, k}\right)\left(-\beta_{p r} T+\Sigma_{p r m n} \in_{m n}+\zeta_{p r s n} a_{s} a_{n}+\kappa_{p r y n} z_{y} z_{n}\right) .
$$

Due to the $\Sigma_{\text {prmn }}=\Sigma_{\text {prnm }}$ symmetry property of the coefficient $\Sigma_{\text {prmn }}$ in this expression, the constitutive equation given by expression (5.13) can be converted into the following form in terms of linear constituents of the displacement gradient:

$$
\begin{aligned}
t_{p r}= & \Gamma_{a} a_{p} a_{r}+\Gamma_{z} z_{p} z_{r}-\beta_{p r} T+\Sigma_{p r m n} u_{m, n}+\zeta_{p r s n} a_{s} a_{n}+\kappa_{p r y n} z_{y} z_{n} \\
& -\zeta_{p r s n} a_{s} a_{n} u_{k, k}-\kappa_{\text {pryn }} z_{y} z_{n} u_{k, k} .
\end{aligned}
$$

In a composite material reinforced by two arbitrary independent and inextensible fiber families, the medium is assumed to be anisotropic, compressible, homogeneous, dependent on temperature gradient and showing linear elastic behavior. Equation (5.14) is the linear constitutive equation of stress. First and second terms on the right part of (5.14) are caused by the inextensibility of the fibers. $\Gamma_{a}$ and $\Gamma_{z}$-fiber stretch, both are determined through field equations and boundary conditions. These two terms are reaction stresses and cannot be expressed by any constitutive equation. The third term expresses the temperature effect, and the fourth term expresses the contribution of the elastic deformation to the stress. Regarding the fifth and the sixth term, two interpretations are possible. The first one states that if the medium is not loaded in any way $\left(T=\right.$ constant, $\left.E_{K L}=0\right)$, it will not switch to stress and therefore physically it should be $\zeta_{\text {prsn }}=0$ and $\kappa_{\text {pryn }}=0$, because being loaded with fibers is not sufficient for a medium to automatically get stressed. Another interpretation can be as follows. No parameters have been used related with cross-section thickness of fibers neither in this study nor in other studies examining macroscopic behavior of fiber-reinforced media. In other words, distribution of fibers is present in the medium only as a topologic object that just causes anisotropy, which means it is completely geometric. In this regard, there is no constraint that would prevent us from reinforcing fiber on the molecular scale. Therefore, if a distribution can be practically placed into the medium in the form of a molecular chain, it 
is possible to suggest that this will alter the present ionic distribution and stress the medium with no other effect. In this case, coefficients $\zeta_{\text {prsn }}$ and $\kappa_{\text {pryn }}$ in fifth and sixth terms will be different from zero and will thus gain a physical meaning. These terms can be interpreted as internal stress contribution stimulated by dislocation. The seventh and eighth terms show the stress formed by interaction of the deformation field with contributions of fiber fields.

If it is assumed that the medium is without fibers, expression (5.14) will be reduced to retain the third and fourth terms indicating the contribution to the stress of the temperature and strain tensor. Accordingly, in this study, terms of (5.14) have been obtained under the mentioned assumptions and are reduced to generally known classical expressions in special cases. This supports the opinion proving the reliability of the model we have created. These new terms are the expressions of constitutive equation on spatial coordinates for stress on a mathematical model created for fabricated composites, specifically for materials involving a distribution of two absolutely arbitrary fibers.

\section{Determination of Heat Flux Vector Constitutive Equation in Linear Thermoelasticity}

Here, the approach assumed for the stress potential has been assumed for the heat flux vector. Accordingly, the heat flux vector can be found through a power series expansion in terms of components of the arguments on which it depends, around a reference location selected as the natural condition. Considering that $\mathbf{E}$ can be substituted by $\widetilde{\mathbf{E}}$ in the linear theory, arguments on which the heat flux vector depends, entropy inequality, and constraint caused by this inequality have been found as follows:

$$
\begin{aligned}
& Q_{R}=Q_{R}(\underline{\underline{\tilde{E}}}, \underline{A}, \underline{Z}, \underline{G}, \theta, \underline{X}), \\
& \mathbf{Q}(\widetilde{\mathbf{E}}, \mathbf{A}, \mathbf{Z}, \mathbf{G}, \theta, \mathbf{X}) \cdot \mathbf{G} \geq 0, \\
& Q_{R}=Q_{R}(\widetilde{\mathbf{E}}, \mathbf{A}, \mathbf{Z},, 0, \theta, \mathbf{X})=0 .
\end{aligned}
$$

Expanding the function (6.1) in a Taylor series around $\widetilde{\mathbf{E}}=\mathbf{0}, \mathbf{A}=\mathbf{0}, \mathbf{Z}=\mathbf{0}, \mathbf{G}=\mathbf{0}$ will give us the following expression:

$$
\begin{aligned}
Q_{R}(\widetilde{\mathbf{E}}, \mathbf{A}, \mathbf{Z}, \mathbf{G}, \theta, \mathbf{X})= & Q_{R}(\mathbf{G}, \theta, \mathbf{X})+\left.\frac{\partial Q_{R}(\widetilde{\mathbf{E}}, \mathbf{G}, \theta, \mathbf{X})}{\partial E_{L M}}\right|_{\mathrm{E}=0} \widetilde{E}_{L M} \\
& +\left.\frac{\partial Q_{R}(\mathbf{A}, \mathbf{G}, \theta, \mathbf{X})}{\partial A_{L} A_{M}}\right|_{\mathbf{A}=0} A_{L} A_{M}+\left.\frac{\partial Q_{R}(\mathbf{Z}, \mathbf{G}, \theta, \mathbf{X})}{\partial Z_{L} Z_{M}}\right|_{\mathbf{Z}=0} Z_{L} Z_{M} .
\end{aligned}
$$


Here, the definitions as in the following are used

$$
\begin{gathered}
Q_{R}(\mathbf{G}, \theta, \mathbf{X}) \equiv Q_{R}(\theta, \mathbf{X})+\left.\frac{\partial Q_{R}(\mathbf{G}, \theta, \mathbf{X})}{\partial G_{L}}\right|_{\mathbf{G}=\mathbf{0}} G_{L}=B_{R}(\theta, \mathbf{X})+B_{R L}(\theta, \mathbf{X}) G_{L}+\cdots \\
\left.H_{R L M}(\mathbf{G}, \theta, \mathbf{X}) \equiv \frac{\partial Q_{R}(\widetilde{\mathbf{E}}, \mathbf{G}, \theta, \mathbf{X})}{\partial E_{L M}}\right|_{\mathbf{E}=\mathbf{0}}=B_{R L M}(\theta, \mathbf{X})+\cdots \\
\left.Y_{R L M}(\mathbf{G}, \theta, \mathbf{X}) \equiv \frac{\partial Q_{R}(\mathbf{A}, \mathbf{G}, \theta, \mathbf{X})}{\partial A_{L} A_{M}}\right|_{\mathbf{A}=0}=D_{R L M}(\theta, \mathbf{X})+\cdots \\
\left.N_{R L M}(\mathbf{G}, \theta, \mathbf{X}) \equiv \frac{\partial Q_{R}(\mathbf{Z}, \mathbf{G}, \theta, \mathbf{X})}{\partial Z_{L} Z_{M}}\right|_{\mathbf{Z}=\mathbf{0}}=F_{R L M}(\theta, \mathbf{X})+\cdots
\end{gathered}
$$

Using the above-mentioned definitions in the series expansion given by expression (6.4) can give us the following expression:

$$
\begin{aligned}
Q_{R}(\widetilde{\mathbf{E}}, \mathbf{A}, \mathbf{Z}, \mathbf{G}, \theta, \mathbf{X})= & B_{R}(\theta, \mathbf{X})+B_{R L}(\theta, \mathbf{X}) G_{L}+B_{R L M}(\theta, \mathbf{X}) \widetilde{E}_{L M} \\
& +D_{R L M}(\theta, \mathbf{X}) A_{L} A_{M}+F_{R L M} Z_{L} Z_{M}+\cdots
\end{aligned}
$$

Due to the symmetry of the tensor $\widetilde{\mathbf{E}}$ and independence of derivatives in the definitions in expressions (6.5) from the order, these coefficients bear the symmetry characteristics given below:

$$
B_{R L M}=B_{R M L}, \quad D_{R L M}=D_{R M L}, \quad F_{R L M}=F_{R M L} .
$$

Since $\mathbf{G}=\mathbf{0} \Rightarrow \mathbf{Q}=\mathbf{0}$ due to the constraint in (6.3), the following expression can be written down from the relation (6.6):

$$
\underline{0}=B_{R}(\theta, \mathbf{X})+B_{R L M}(\theta, \mathbf{X}) \tilde{E}_{L M}+D_{R L M}(\theta, \mathbf{X}) A_{L} A_{M}+F_{R L M}(\theta, \mathbf{X}) Z_{L} Z_{M}+\cdots
$$

Since expression (6.8) is zero for any arbitrary deformation measure, coefficients in this equation should be zero. Therefore,

$$
B_{R}(\theta, \mathbf{X})=B_{R L M}(\theta, \mathbf{X})=D_{R L M}(\theta, \mathbf{X})=F_{R L M}(\theta, \mathbf{X})=0 .
$$

Accordingly, (6.6) is reduced to the following form:

$$
Q_{R}=Q_{R}(\widetilde{\mathbf{E}}, \mathbf{A}, \mathbf{Z}, \mathbf{G}, \theta, \mathbf{X})=B_{R L}(\theta, \mathbf{X}) G_{L}=B_{R L}(\theta, \mathbf{X}) \theta,{ }_{L}
$$

Substituting expression (6.10) in the inequality (6.2) gives us the following expression:

$$
B_{R L}(\theta, \mathbf{X}) \theta,{ }_{L} \theta,{ }_{R} \geq 0 \quad \text { or } B_{R L}(\theta, \mathbf{X}) G_{L} G_{R} \geq 0 \text {. }
$$


Therefore, the tensor $B_{R L}(\theta, \mathbf{X})$ should satisfy the following condition for any temperature gradient:

$$
B_{R L} \theta_{, R} \theta_{, L} \geq 0 \quad \text { or } B_{(R L)} \theta_{, R} \theta_{, L} \geq 0
$$

The $B_{R L}$ tensor is named conductivity coefficient tensor. Inequality (6.12) tells us that the symmetric part of this tensor is positive definite. For the linear theory, the coefficient $B_{R L}$ is expressed as follows in similarity to the coefficient $\Sigma_{P R}$ :

$$
B_{R L}(\theta, \mathbf{X})=B_{R L}\left(T_{0}+T, \mathbf{X}\right)=B_{R L}\left(T_{0}, \mathbf{X}\right)+\left.\frac{\partial B_{R L}(T, \mathbf{X})}{\partial T}\right|_{0} T+\cdots
$$

Moreover, the coefficient $\theta_{, L}$ can be written down as follows:

$$
\theta_{, L}=\left(T_{0}+T\right)_{, L}=T_{, L}
$$

Substituting expression (6.14) in (6.10) and omitting the nonlinear term $(T)\left(T_{, L}\right)$, the heat flux vector is written down as follows:

$$
Q_{R}=B_{R L}\left(T_{0}, \mathbf{X}\right) T_{, L} .
$$

The expression of the spatial form of heat flux vector for compressible media can be written down as indicated below:

$$
q_{r}=\left(1-u_{k, k}\right) Q_{R} x_{r, R}
$$

If (6.15) is substituted into expression (6.16), using expressions (5.5) and omitting the nonlinear term $\left(u_{k, k} T_{l,}\right)$, the spatial form of the heat flux vector follows as

$$
q_{r}=B_{r l}\left(T_{0}, \mathbf{X}\right) T_{, l} .
$$

The spatial tensor $B_{r l}$ of the material in (6.17) has the same symmetry characteristics as the tensor $B_{R L}$ and is defined as follows:

$$
B_{r l} \equiv \lambda_{r R} \lambda_{l L} B_{R L} .
$$

Equation (6.17) is the Fourier heat transfer law, which defines linear heat transfer, and it can be written down as follows in the vectorial form:

$$
\mathbf{q}=\mathbf{B} \nabla T
$$




\section{Determination of Field Equations}

Before proceeding to obtain the field equations, let us discuss the meaning of the tensor $\beta_{p r}$ in (5.14). Firstly, let us define the tensor $\Sigma_{p r m n}^{-1}$, which is the reversed tensor $\Sigma_{p r m n}$ and has the same symmetry properties as this tensor, as follows:

$$
\Sigma_{p r m n} \Sigma_{m n k l}^{-1} \equiv \frac{1}{2}\left(\delta_{p k} \delta_{r l}+\delta_{p l} \delta_{r k}\right), \quad \Sigma_{p r m n}^{-1}=\Sigma_{r p m n}^{-1}=\Sigma_{m n p r}^{-1}=\Sigma_{p r n m}^{-1}
$$

The tensor $\alpha_{p r}$ comprised of thermal expansion coefficients that can be easily measured physically can be defined as follows:

$$
\alpha_{p r} \equiv \Sigma_{p r m n}^{-1} \beta_{m n}=\alpha_{r p}
$$

To find the reverse of expression (7.2), let us multiply both sides of the equation by the tensor $\Sigma_{k l p r}$. Then, using a suitable index replacement, the following can be written down:

$$
\beta_{p r}=\Sigma_{p r m n} \alpha_{m n}
$$

Substituting expression (7.3) in (5.14) will give us the following:

$$
\begin{aligned}
t_{p r}= & \Gamma_{a} a_{p} a_{r}+\Gamma_{z} z_{p} z_{r}+\zeta_{p r s n}\left(a_{s} a_{n}-u_{k, k} a_{s} a_{n}\right)+\kappa_{p r y n}\left(z_{y} z_{n}-u_{k, k} z_{y} z_{n}\right) \\
& +\Sigma_{p r m n}\left(u_{m, n}-\alpha_{m n} T\right) .
\end{aligned}
$$

The following expression can be written down in regard to a linear theory:

$$
\rho \dot{v}_{k} \cong \rho_{0}\left(1-u_{k, k}\right) \frac{\partial v_{k}}{\partial t} \cong \rho_{0} \frac{\partial}{\partial t} \frac{\partial u_{k}}{\partial t} \cong \rho_{0} \frac{\partial^{2} u_{k}}{\partial t^{2}}
$$

In a linear theory, the Cauchy equations of motion can be written as follows substituting the expressions (7.5) and (5.5) in (3.2):

$$
t_{k l, l}+\rho_{0}\left(1-u_{l, l}\right) \mathrm{f}_{\mathrm{k}}=\rho_{0} \frac{\partial^{2} u_{k}}{\partial t^{2}} .
$$

Considering that the medium is homogenous and omitting the term $\left(\rho_{0} u_{l, l} \mathrm{f}_{\mathrm{k}}\right)$, let us calculate the divergence of the stress given by (7.4) and substitute it in (7.6) to obtain the following field equation under the above-mentioned assumptions:

$$
\begin{aligned}
\rho_{0} \frac{\partial^{2} u_{p}}{\partial t^{2}}= & \Sigma_{p r m n}\left(u_{m, n r}-\alpha_{m n} T_{, r}\right)+\rho_{0} \mathrm{f}_{\mathrm{p}}+\left(\Gamma_{a}\right)_{, r} a_{p} a_{r}+\Gamma_{a}\left(a_{p, r} a_{r}+a_{p} a_{r, r}\right) \\
& +\left(\Gamma_{z}\right)_{, r} z_{p} z_{r}+\Gamma_{z}\left(z_{p, r} z_{r}+z_{p} z_{r, r}\right)+\zeta_{p r s n}\left(a_{s, r} a_{n}+a_{s} a_{n, r}\right) \\
& -\zeta_{p r s n}\left(a_{s, r} a_{n}+a_{s} a_{n, r}\right) u_{k, k}-\zeta_{p r s n} u_{k, k r} a_{s} a_{n}+\kappa_{p r y n}\left(z_{y, r} z_{n}+z_{y} z_{n, r}\right) \\
& -\kappa_{\text {pryn }}\left(z_{y, r} z_{n}+z_{y} z_{n, r}\right) u_{k, k}-\kappa_{p r y n} u_{k, k r} z_{y} z_{n} .
\end{aligned}
$$


Expression (7.7) gives us a field equation with the unknowns $u_{k}, \Gamma_{a}, \Gamma_{z}$. The solution of this field equation under initial and boundary conditions forms the mathematical structure of a boundary value problem to consider.

Because $\theta=T_{0}+T$ and $\partial T / \partial \theta=1$, the entropy and the internal energy density given in expressions (4.15) and (4.22) can be written down as follows:

$$
\begin{aligned}
& \eta=-\frac{1}{\rho_{0}} \frac{\partial \Sigma}{\partial T} \frac{\partial T}{\partial \theta}=-\frac{1}{\rho_{0}} \frac{\partial \Sigma}{\partial T} \\
& \varepsilon=\frac{1}{\rho_{0}}\left[\Sigma-\left(T_{0}+T\right) \frac{\partial \Sigma}{\partial T}\right] .
\end{aligned}
$$

Substituting (7.8) in expression (7.9) will give us the following expression:

$$
\varepsilon=\frac{\Sigma}{\rho_{0}}+\left(T_{0}+T\right) \eta
$$

Taking the derivative of $\Sigma$ given by expression (5.12) according to $T$ and substituting it in (7.8) after related operations will allow us to express entropy in terms of the displacement gradient component as follows:

$$
\eta=\eta_{0}+\frac{c T}{T_{0}}+\frac{\beta_{k l}}{\rho_{0}} u_{k, l}+\frac{\mu_{s n}}{\rho_{0}} a_{s} a_{n}+\frac{\pi_{y n}}{\rho_{0}} z_{y} z_{n}
$$

Let us now substitute expressions (5.12) and (7.11) in (7.10) and make necessary arrangements to obtain the internal energy density as follows:

$$
\begin{aligned}
\varepsilon= & \varepsilon_{0}+c\left(T+\frac{T^{2}}{2 T_{0}}\right)+\frac{T_{0} \beta_{k l}}{\rho_{0}} u_{k, l}+\frac{1}{2 \rho_{0}} \Sigma_{k l m n} u_{k, l} u_{m, n} \\
& +\frac{1}{\rho_{0}}\left[\left(\Lambda_{s n}+\zeta_{k l s n} u_{k, l}\right) a_{s} a_{n}+\left(\Omega_{y n}+\kappa_{k l y n} u_{k, l}\right) z_{y} z_{n}\right] .
\end{aligned}
$$

$\varepsilon_{0}$ coefficient in this equation has been defined as $\varepsilon_{0}=\psi_{0}+T_{0} \eta_{0}$, where $\varepsilon_{0}, \psi_{0}$, and $\eta_{0}$ are, respectively, internal energy density, free energy density, and entropy density in natural condition. Taking a material derivative of expression (7.12) and considering that $\rho=\rho_{0}\left(1-u_{k, k}\right)$ give us the following expression:

$$
\begin{aligned}
\rho \dot{\varepsilon}= & \rho_{0}\left(1-u_{m, m}\right) \dot{\varepsilon} \\
= & \rho_{0} c\left(1+\frac{T}{T_{0}}\right) \frac{\partial T}{\partial t}+T_{0} \beta_{k l} \frac{\partial u_{k, l}}{\partial t}+\Sigma_{k l m n} \frac{\partial u_{k, l}}{\partial t} u_{m, n}+\zeta_{k l s n} \frac{\partial u_{k, l}}{\partial t} a_{s} a_{n} \\
& +\kappa_{k l y n} \frac{\partial u_{k, l}}{\partial t} z_{y} z_{n}-\zeta_{k l s n} \frac{\partial u_{k, l}}{\partial t} u_{m, m} a_{s} a_{n}-\kappa_{k l y n} \frac{\partial u_{k, l}}{\partial t} u_{m, m} z_{y} z_{n}
\end{aligned}
$$


The term $q_{r, r}$ is obtained as follows from (6.17):

$$
q_{r, r}=B_{r l, r} T_{l}+B_{(r l)} T_{, l r}=B_{(r l)} T_{, l r}
$$

Let us now substitute the expressions (7.13), (7.14), (7.4), and (5.5) in the equation conservation of energy given by expression (3.4) and make necessary arrangements to make the following field equations linear in terms of $u_{k, l}$ and $T$ :

$$
\rho_{0} c \frac{\partial T}{\partial t}+\left(T_{0} \beta_{k l}-\Gamma_{a} a_{k} a_{l}-\Gamma_{z} z_{k} z_{l}\right) \frac{\partial u_{k, l}}{\partial t}=\beta_{(k l)} T_{, l k}+\rho_{0}\left(1-u_{l, l}\right) h
$$

In a composite material reinforced by two arbitrary independent and inextensible fiber families, where the medium is assumed to be anisotropic, compressible, homogeneous, dependent on temperature gradient, and showing linear elastic behavior, (7.15) is a heat transfer equation.

\section{Conclusions}

As an approach in this study, the stress potential and heat flux vector functions have been assumed to be analytic and expanded in Taylor series in terms of their arguments on which they depend. The type and the number of terms taken in the series expansion have been determined based on the assumption that mechanical interactions and temperature changes are linear. On the other hand, since the matrix material has to remain insensitive to directional changes along fibers, even-numbered exterior products of vector fields representing fiber distributions have been considered. The reference position of the medium has been selected at the uniform temperature $T_{0}$ and stress-free natural condition, from which position the medium has been assumed to move away by small displacements, and small temperature changes. Accordingly, the forms in spatial coordinates of the constitutive equations of the stress and the heat flux vector have been presented by (5.14) and (6.17). The constitutive equation of the stress expressed by (5.14) in terms of the tensor $\alpha_{p r}$ comprised of thermal expansion coefficients has been expressed by (7.4). To obtain field equations, constitutive equation of the stress given by (7.4) has been substituted into the Cauchy equation of motion, yielding field equation (7.7). Values in the equation of conservation of energy given by expression (3.4) have been substituted into (3.4), yielding field equation (7.15). Solution of the field equations along with initial and boundary conditions in conformity with the structure of the problem to be used in practice will constitute the structure of a boundary value problem to consider. Unknowns in the field equations (7.7) and (7.15), $\mathbf{u}(\mathbf{x}, t), \Gamma_{a}$, and $\Gamma_{z} . \Gamma_{a}$ and $\Gamma_{z}$, which are Lagrange coefficients, can be calculated using the field equations and boundary conditions. After $\mathbf{u}$ has been designated, the stress distribution is obtained from (7.4). After the stress distribution is found as a tensor field, the stress vector at a desired cross-section can be easily calculated from the expression $\mathbf{t}_{(\mathbf{n})_{r}}=n_{p} t_{p r}$. Here, it needs to be considered that the fiber distributions $a_{k}(\mathbf{x}, t)$ and $z_{k}(\mathbf{x}, t)$ after deformation for inextensible fibers in terms of fiber distributions before deformation are $a_{k}=x_{k, K} A_{K}(\mathbf{X})$ and $z_{k}=x_{k, K} Z_{K}(\mathbf{X})$.

Besides, considering the equations of motion (7.7), we can see the internal thermomechanical forces affecting the medium. Type of the terms on the right is in the dimension of force per unit of volume. The first term on the right represents force created by the elastic deformation, the second term is force created by the temperature gradient, the third 
term is mechanical body force, and the fourth and the sixth terms are similar to molecular considerations in (5.14). The fifth, seventh, eighth, and eleventh terms are forces caused by curvature of the fibers. The ninth and twelfth terms are forces caused by interaction of fibers and their curvature with the deformation field. The tenth and thirteenth terms represent forces caused by interaction of the deformation field with distributions of fiber fields. In other words, by drawing a free body diagram of a material element in the medium, it is possible to see all such force contributions acting on the element. As stated before, our field equations where we can apply initial and boundary conditions for the aforementioned media are (7.7) and (7.15). A more detailed discussion of these equations will be contained in future works.

\section{Acknowledgments}

The author would like to thank the anonymous referees who made much useful and detailed suggestions that helped her to correct some errors and improve the quality of this paper. The author is also grateful to professor Horst Ecker for his editorial helping and valuable comments which have greatly aided in the improvement of this paper.

\section{References}

[1] M. Usal, A mathematical model for a biological construction element, Ph.D. thesis, Süleyman Demirel University, Institute of Science and Technology, Isparta, Turkey, 2001.

[2] R. M. Jones, Mechanics of Composite Materials, Scripta Book, Washington, DC, USA, 1975.

[3] D. Hull, An Introduction to Composite Materials, Cambridge University Press, Cambridge, UK, 1990.

[4] M. Usal, M. R. Usal, and Ü. Esendemir, "A continuum formulation for fiber-reinforced viscoelastic composite materials with microstructure Part - I: anisotropic matrix material," Science and Engineering of Composite Materials, vol. 15, no. 3, pp. 217-234, 2008.

[5] M. R. Usal, M. Usal, and Ü. Esendemir, "A mathematical model for thermomechanical behavior of arbitrary fiber reinforced viscoelastic composites - I," Science and Engineering of Composite Materials, vol. 13, no. 4, pp. 291-300, 2006.

[6] A. Ü. Erdem, M. R. Usal, and M. Usal, "A mathematical model for the electrothermomechanical behavior of an arbitrarily fiber-reinforced viscoelastic piezoelectric body," Journal of the Faculty of Engineering and Architecture of Gazi University, vol. 20, no. 3, pp. 305-319, 2005.

[7] M. Usal, M. R. Usal, and A. U. Erdem, "On magneto-viscoelastic behavior of fiber-reinforced composite materials part I: anisotropic matrix material," Science and Engineering of Composite Materials, vol. 16, no. 1, pp. 41-56, 2009.

[8] M. R. Usai, "A constitutive formulation of arbitrary fiber-reinforced viscoelastic pieoelectric compsite materials-part one," International Journal of Nonlinear Sciences and Numerical Simulation, vol. 8, no. 2, pp. 257-274, 2007.

[9] G. A. Maugin and A. Berezovski, "Material formulation of finite-strain thermoelasticity and applications," Journal of Thermal Stresses, vol. 22, no. 4-5, pp. 421-449, 1999.

[10] V. K. Kalpakides and C. Dascalu, "On the configurational force balance in thermomechanics," Proceedings of the Royal Society of London. Series A, vol. 458, no. 2028, pp. 3023-3039, 2002.

[11] V. A. Lubarda, “On thermodynamic potentials in linear thermoelasticity," International Journal of Solids and Structures, vol. 41, no. 26, pp. 7377-7398, 2004.

[12] W. Nowacki, Dynamic Problems of Thermoelasticity, Noordhoff International, The Netherlands, 1975.

[13] G. Karami and M. Garnich, "Micromechanical study of thermoelastic behavior of composites with periodic fiber waviness," Composites Part B, vol. 36, no. 3, pp. 241-248, 2005.

[14] A. Maewal and D. P. Dandekar, "Effective thermoelastic properties of short-fiber composites," Acta Mechanica, vol. 66, no. 1-4, pp. 191-204, 1987.

[15] J.-S. Chang and C.-H. Cheng, "Thermoelastic properties of short-coated fiber composites: effects of length and orientation distributions," Composites Science and Technology, vol. 55, no. 4, pp. 329-341, 1995. 
[16] M. Gorji and F. Mirzadeh, "Theoretical prediction of the thermoelastic properties and thermal stresses in unidirectional composites," Journal of Reinforced Plastics and Composites, vol. 8, no. 3, pp. 232-258, 1989.

[17] S. K. Kanaun and V. M. Levin, "Elastic and thermoelastic properties of composites reinforced with short axisymmetric fibers," International Journal of Damage Mechanics, vol. 4, no. 3, pp. 264-282, 1995.

[18] H.-J. Chun, J.-Y. Shin, and I. M. Daniel, "Effects of material and geometric nonlinearities on the tensile and compressive behavior of composite materials with fiber waviness," Composites Science and Technology, vol. 61, no. 1, pp. 125-134, 2001.

[19] V. P. Privalko, D. I. Sukhorukov, and J. Karger-Kocsis, "Thermoelastic behavior of carbon fiber/polycarbonate model composites," Polymer Engineering and Science, vol. 39, no. 8, pp. 1525-1533, 1999.

[20] K. Honjo, "Thermal stresses and effective properties calculated for fiber composites using actual cylindrically-anisotropic properties of interfacial carbon coating," Carbon, vol. 45, no. 4, pp. 865-872, 2007.

[21] B. Nedjar, "An anisotropic viscoelastic fibre-matrix model at finite strains: continuum formulation and computational aspects," Computer Methods in Applied Mechanics and Engineering, vol. 196, no. 912, pp. 1745-1756, 2007.

[22] E. S. Şuhubi, Continuum Mechanics: Introduction, İ.T.U., Faculty of Arts and Sciences Publication, İstanbul, Turkey, 1994.

[23] A. J. M. Spencer, Continuum Theory of the Mechanics of Fibre Reinforced Composites, Cism International center for mechanical sciences, Course and lecturers, A. J. M. Spencer Eds.,, Springer, New York, NY, USA, 1984.

[24] A. J. M. Spencer, Deformations of Fibre-Reinforced Materials, Clarendon Press, Oxford, UK, 1972.

[25] A. C. Eringen, Mechanics of Continua, Robert E. Krieger Publishing, Hungtington, NY, USA, 1980.

[26] B. Hamamc1, A mathematical model for fiber reinforced thermoelastic materials, M.S. thesis, Süleyman Demirel University, Institute of Science and Technology, Isparta, Turkey, 2006.

[27] M. R. Usal, A mathematical model for the electro-thermomechanical behaviour of fiber reinforced elastic dielectric media, Ph.D. thesis, Erciyes University, Institute of Science and Technology, Kayseri, Turkey, 1994. 


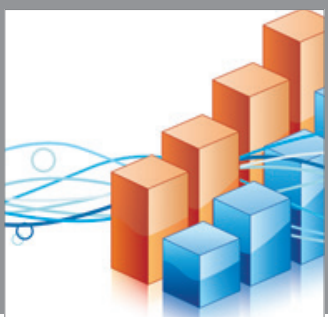

Advances in

Operations Research

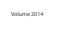

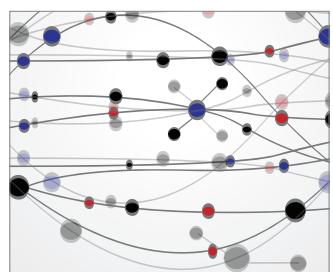

\section{The Scientific} World Journal
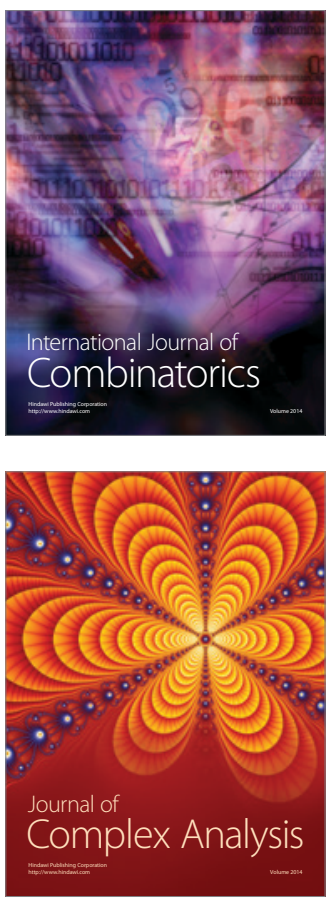

International Journal of

Mathematics and

Mathematical

Sciences
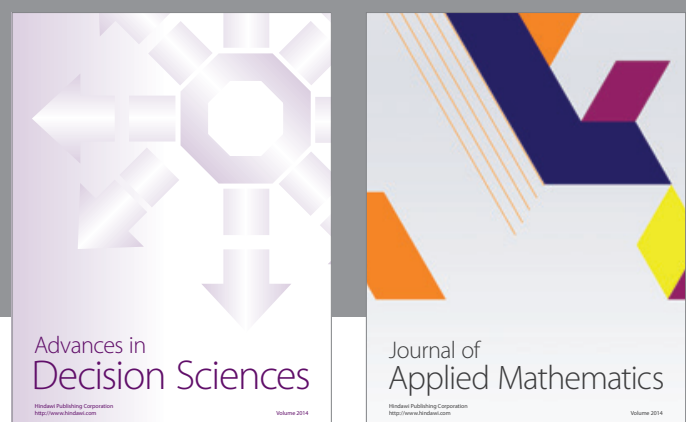

Journal of

Applied Mathematics
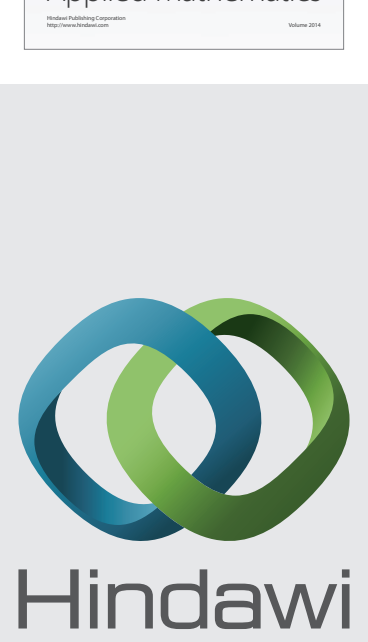

Submit your manuscripts at http://www.hindawi.com
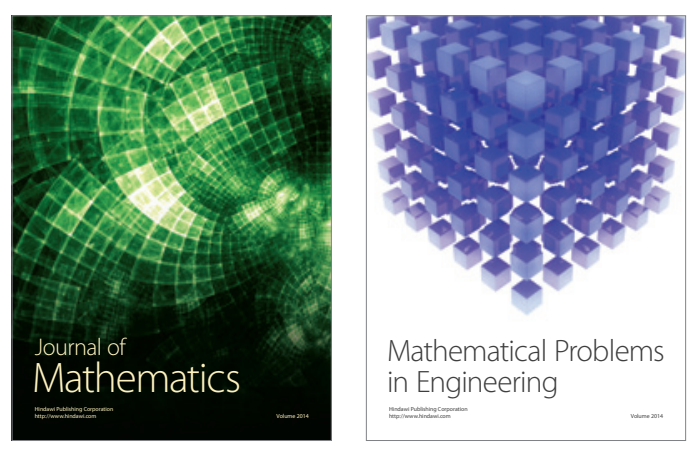

Mathematical Problems in Engineering
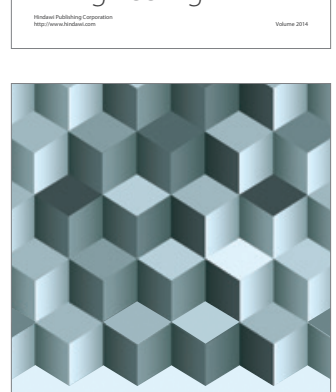

Journal of

Function Spaces
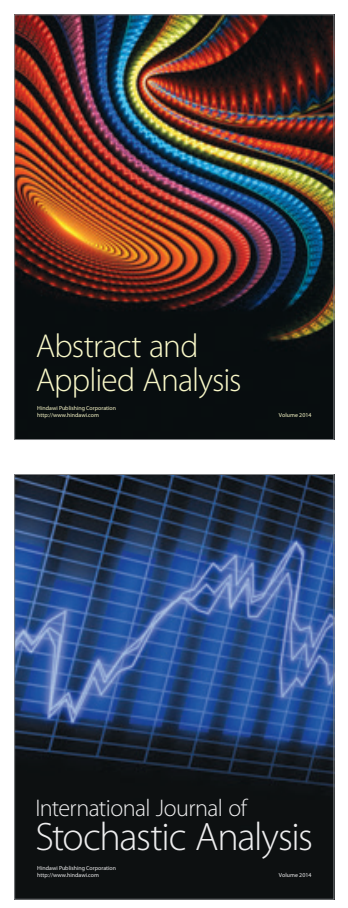

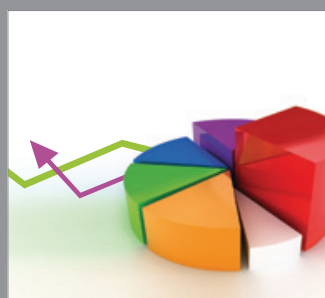

ournal of

Probability and Statistics

Promensencen
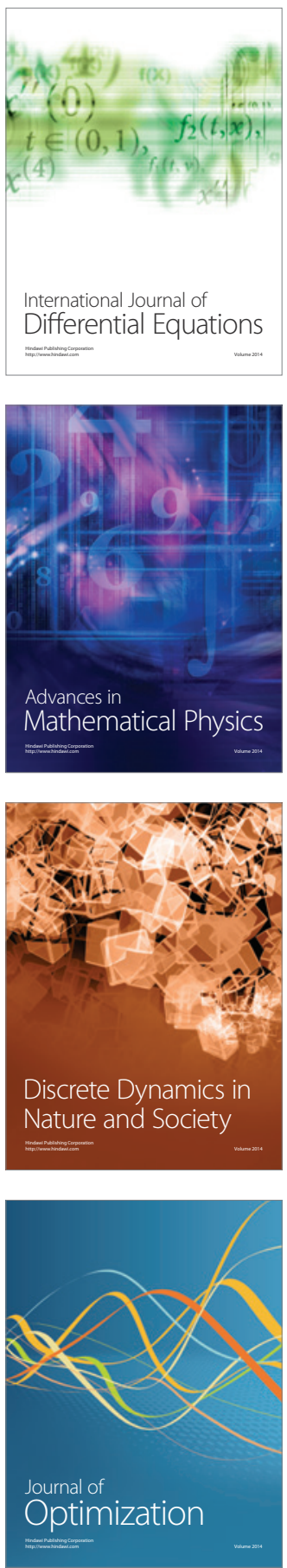